\title{
A rare case of successful reproductive outcome after IVF following endometrioma resection in a patient of arcuate uterine anomaly and urinary bladder wall endometrioma in women with primary infertility
}

\author{
Anju Dab $^{1 *}$, Jigna Garasia ${ }^{1}$, Pooja Singh ${ }^{2}$, Purnima Nadkarni ${ }^{1}$
}

\begin{abstract}
${ }^{1}$ Department of Department of Reproductive Medicine, Nadkarni's Test Tube Baby Centre, Killa Pardi, Gujarat, India ${ }^{2}$ Department of Department of Reproductive Medicine, Nadkarni's $21^{\text {st }}$ Century Hospital and Test Tube Baby Centre, Surat, Gujarat, India
\end{abstract}

Received: 23 September 2016

Accepted: 20 October 2016

\section{*Correspondence:}

Dr. Anju Dab,

E-mail: dranjudab@gmail.com

Copyright: $\odot$ the author(s), publisher and licensee Medip Academy. This is an open-access article distributed under the terms of the Creative Commons Attribution Non-Commercial License, which permits unrestricted non-commercial use, distribution, and reproduction in any medium, provided the original work is properly cited.

\begin{abstract}
Endometriosis is a common gynecologic disease in women of reproductive age. However, endometriosis within the bladder is rare. We present a rare case of a 38-year-old woman who presented with dysmenorrhea and primary infertility. Transvaginal ultrasonography showed an arcuate bulky adenomyotic uterus and left ovarian cystic mass along with urinary bladder endometrioma. Cystoscopic examination was done and removal of endometrioma was attempted, which failed. Afterwards laparoscopic exploration of abdomen was done and a partial cystectomy was performed and it was confirmed that the mass on the bladder wall was a $3.5 \mathrm{~cm}$ endometrioma invading the vesical wall on the final pathologic report. Post-operatively, she underwent in vitro fertilization due to both causes i.e. primary infertility and male factor (severe oligo-asthenospermia) and she conceived. We report this case of a patient with primary infertility and bladder endometriosis which was managed successfully with a surgical approach and briefly review the relevant literature.
\end{abstract}

Keywords: Bladder endometriosis, In vitro fertilization

\section{INTRODUCTION}

Endometriosis is a common benign gynaecological disease characterized by the presence of ectopic endometrial tissue, outside the uterus. The major clinical manifestations of endometriosis are cyclical hematuria, chronic pelvic pain and infertility. ${ }^{1}$ The common sites of endometriosis are the ovaries, posterior cul-de-sac, posterior broad ligaments, uterosacral ligaments, uterus, and fallopian tubes. ${ }^{2}$ Endometriosis with bladder involvement is a rare condition, even though the bladder is the most often affected organ among the urinary tract structures. The incidence of bladder endometriosis is reported to be $<1 \%$ of all types of endometriosis. ${ }^{1}$ Although endometriosis is a benign disease, it has the capacity to grow, infiltrate, and disseminate in a malignancy like manner. ${ }^{3}$
Therefore, complete surgical excision is essential in the management of endometriosis. We report a patient with primary infertility and bladder endometriosis that was managed successfully by surgical approach and pregnancy was achieved using assisted reproductive techniques.

\section{CASE REPORT}

A 38 year-old, married nulligravida presented for evaluation of severe dysmenorrhoea of 8 months duration, beginning in January 2016 and primary infertility, being married for 21 years. Her symptoms were more severe after menses. She had regular menstrual cycles, 25 days in length, with menstruation lasting 3 4 days and moderate menstrual bleeding along with cyclical hematuria. She had severe dysmenorrhoea, 
which had worsened since 2014, so she usually took over the counter pain medications for relief of symptoms. She denied flank pain or a history of nephrolithiasis. She was married for 21 years and has unsuccessfully tried to conceive.

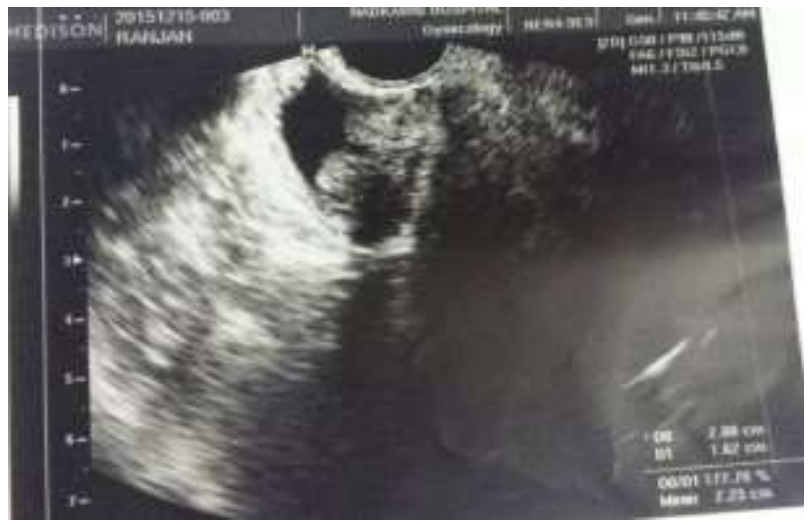

Figure 1: Abdominal sonography shows intravesical low echogenic mass.

The pelvic examination revealed no palpable masses. The laboratory examination revealed the following: white blood count, 4,600/ $/ \mathrm{mm}^{3}$; haemoglobin, $11.7 \mathrm{~g} / \mathrm{dL}$; platelet count, $157,000 / \mathrm{mm}^{3}$; serum creatinine, 0.7 $\mathrm{mg} / \mathrm{dL}$; and $\mathrm{CA}-125,18.8 \mathrm{U} / \mathrm{m}$. The other laboratory findings were in the normal range. Ultrasound (transabdominal and endovaginal) showed an iso to hyper-echoic mass with a smooth surface and few cystic areas along the posterior wall of the urinary bladder appearing to protrude into its lumen. Cystoscopy identified a mass on the dome of bladder. The patient underwent laparoscopic exploration of abdomen. The intra-operative findings included bilateral tubal obstruction, posterior cul-de-sac obliteration, and severe adhesions between the uterus and bladder. Overall, the structure of pelvic organs was distorted by severe adhesions. After careful adhesiolysis was performed, the bladder mass was identified. A $3.5 \mathrm{~cm}$ mass consisting of confluent nodules and a hemorrhagic cyst was found on the wall of the bladder. This mass was removed with help of laproscopic intervention and partial cystectomy was done. Afterwards, D-J stenting of left ureter was done, which was removed after 21 days. It was confirmed that the mass on the bladder wall was a $3.5-\mathrm{cm}$ endometrioma invading the vesical wall on the final histopathology report (Figure 2 and Figure 3). She was discharged on post-operative day 7 without complications. After we confirmed no urinary leakage on cystography, Foley's catheter was removed on post operative day 14.

After foley`s catheter removal, she had no discomfort, except for urinary frequency due to a decreased bladder volume. She was put on long acting LHRH agonist Zoladex $3.60 \mathrm{mg}$ (Goserelin acetate) followed up by 3 months once daily treatment with Dienogest $2 \mathrm{mg}$ (steroidal progestin of the 19-nortestosterone group). After this her endometrial study and ovum donation was done. She was then attempted Intracytoplasmic sperm injection with her husband's sperm on donated ovum in March 2016 and achieved a pregnancy. The expected date of confinement is 21 December 2016 and she had enjoyed a healthy pregnancy without complications thus far.

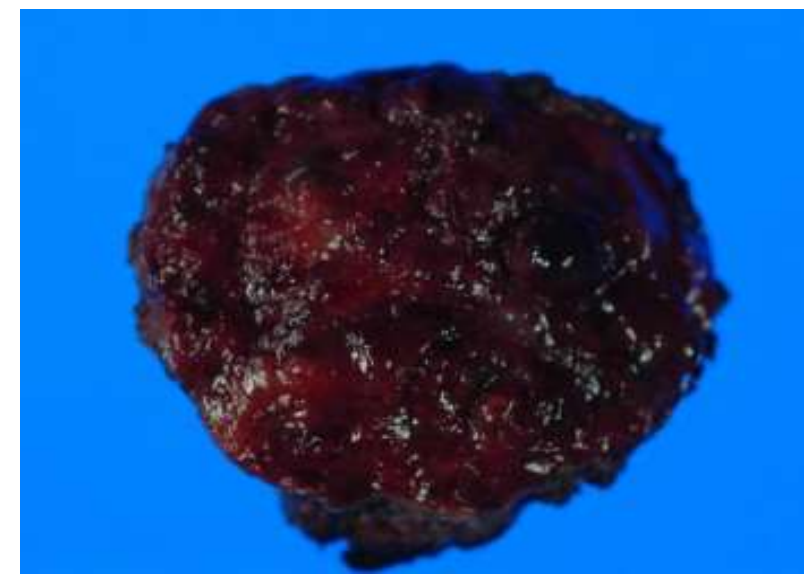

Figure 2: Gross image of the specimen shows a $3.5 \mathrm{~cm}$ hemorrhagic mass on the bladder wall.

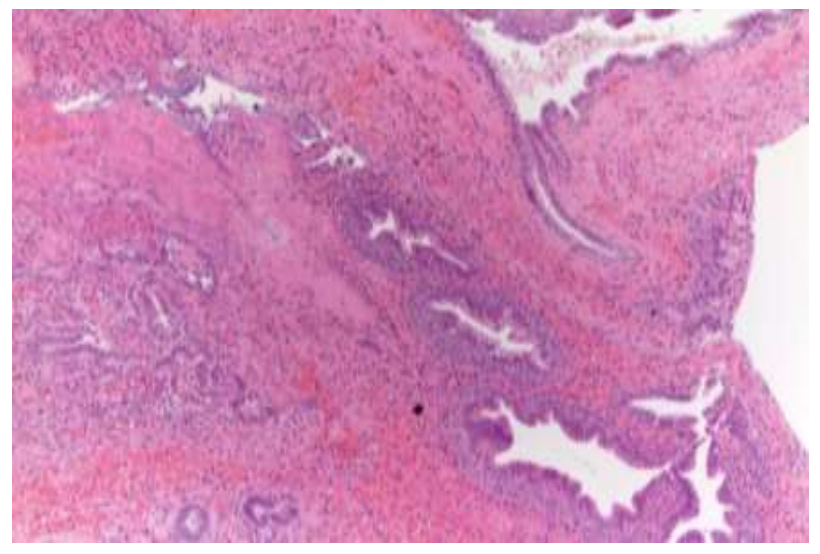

Figure 3: Microscopic finding of the specimen shows endometrial glands and stroma in the bladder muscle (H and E stain, $x$ 10).

\section{DISCUSSION}

Endometriosis can occur among any women of reproductive age, and more frequently in the 2nd and 3rd decades of life. The estimated prevalence of endometriosis is $3 \sim 10 \%$ of women between 20 and 45 years of age, and 25 35\% of infertile women. ${ }^{2}$ Bladder endometriosis is a very rare condition. Since the first case was reported by Judd in 1921, the incidence of bladder involvement occurs in $<1 \%$ of all endometriosis cases according to several reviews. ${ }^{1,4}$ Three pathogenic hypotheses for bladder endometriosis have been proposed, as follows: trans tubal menstrual reflux of endometrial cells with implantation on the peritoneum covering the bladder dome; extension of adenomyosis from the anterior uterine wall to the bladder; and 
metaplasia of subperitoneal Mullerian remnants located in the vesicovaginal septum. ${ }^{5}$

Majority of the gynaecologists agree to a composite theory of endometrial migration, including retrograde menstruation, lymphovascular metastases, and direct extension of the lesions. ${ }^{6}$ Henriksen suggested that the cause of bladder endometriosis is divided into two forms. ${ }^{7}$ The primary form is a spontaneously occurring manifestation of generalized pelvic disease. The secondary form is iatrogenic, occurring after pelvic surgery, such as caesarean delivery or hysterectomy. After iatrogenic dissemination, growth of ectopic endometrium is usually confined to the bladder wall. Approximately $50 \%$ of patients with bladder endometriosis have a history of gynaecologic or obstetric surgery. $^{2}$

It is postulated that disruption of the uterus produces seeding of endometrial tissue and subsequent infiltration of the surrounding bladder, detrusor, and mucosa. ${ }^{6}$ Seeding of the endometriotic tissue from an endometrioma to the bladder could occur during surgery. Endometriosis of the bladder usually manifests as acute urinary symptoms, such as dysuria, frequency, hematuria, tenesmus, a burning sensation, suprapubic discomfort, and cyclic pain. The presence of cyclic hematuria with these symptoms is considered pathognomonic, yet appearing in only 20 25\% of patients when the bladder mucosa is involved. ${ }^{2}$ In this case patient was not having any micturion problems or hematuria. For the diagnosis of bladder endometriosis, radiographic imaging can demonstrate the size and location of the mass.

Generally, sonography is the initial study distinguishing between solid and cystic lesions. Because CT provides limited information in bladder endometriosis, MRI is a useful and valuable test in identifying the characteristic features of bladder endometriosis allowing evaluation of the extent of the disease within the vesical wall and other associated structures. ${ }^{8}$

When bladder endometriosis is suspected, the differential diagnosis should include bladder carcinomas, angiomas, leiomyomas, and cystopathies (amyloidosis, malakoplakia, glandular cystitis, and nephrogenic adenoma); histologic confirmation is necessary in nearly all cases. The confirmative test for bladder endometriosis is cystoscopy. The cystoscopic image of endometriosis changes over the menstrual cycle, being larger and more congestive during menstruation. ${ }^{2}$

Following cystoscopy, histological confirmation is essential in the diagnosis of bladder endometriosis. The treatment of bladder endometriosis should be decided based on the patient's age, desire for future pregnancies, severity of the clinical symptoms, location of the lesion, and other involved organs. ${ }^{1}$ Most gynaecologists agree that medical treatment alone does not lead to the definitive cure of deep, severe endometriosis. Furthermore, recurrence of pelvic endometriosis is as high as $56 \%$ after medical treatment. ${ }^{2,9,10}$ We suggest that bladder endometriosis should be considered in women of reproductive age who present with cyclic urinary symptoms during menstruation and surgical treatment offers the best approach for the diagnosis and effective management.

\section{Funding: No funding sources \\ Conflict of interest: None declared \\ Ethical approval: Not required}

\section{REFERENCES}

1. Giudice LC, Kao LC. Endometriosis. Lancet. 2004;364:1789-99.

2. Pastor-Navarro H, Gimenez-Bachs JM, DonateMoreno MJ. Update on the diagnosis and treatment of bladder endo-metriosis. Int Urogynecol J Pelvic Floor Dysfunct. 2007;18:949-54.

3. Swiersz LM. Role of endometriosis in cancer and tumor development. Ann N Y Acad Sci. 2002;955:281-92.

4. Judd ES. Adenomyoma presenting as a tumor of the bladder. Surg Clin North Am. 1921;1:1271-3.

5. Neahat CH, Malik S, Osias J, Nezhat F, Nezhet C. Laparo-scopic management of 15 patients with infiltrating endome-triosis of the bladder and a case of a primary intravesical endo-metrioid adenosarcoma. Fertil Steril. 2002;78:872-5.

6. Comiter CV. Endometriosis of the urinary tract. Urol Clin North Am. 2002;29:625-35.

7. Henriksen E. Primary endometriosis of the urinary bladder. JAMA. 1935;104:1401.

8. Umaria N, Olliff JF. MRI appearances of bladder endo-metriosis. Br J Radiol. 2000;73:733-6.

9. Chapron C, Bourret A, Chopin N. Surgery for bladder endometriosis: long-term results and concomitant management of associated posterior deep lesions. Hum Reprod. 2010;25:884-9.

10. Lee SH, Kim SR, Jeon GH. Three cases of endometriosis of the urinary tract. Korean J Obstet Gynecol. 2010;53:83-9.

Cite this article as: Dab A, Garasia J, Singh P, Nadkarni P. A rare case of successful reproductive outcome after IVF following endometrioma resection in a patient of arcuate uterine anomaly and urinary bladder wall endometrioma in women with primary infertility. Int J Reprod Contracept Obstet Gynecol 2016;5:4493-5. 